\title{
Dispersion of chromia films (eskolaite) in UV-VIS
}

\author{
Aarne Kasikov*, Aivar Tarre**, Margus Marandi ${ }^{* *}$
}

\begin{abstract}
Atomic layer deposited polycrystalline $\mathrm{Cr}_{2} \mathrm{O}_{3}$ films grown from chromyl chloride and methanol were analysed using spectrophotometry, spectral ellipsometry and atomic force microscopy. The films possessed polycrystalline eskolaite structure with rough sublayer in contact with air. Using the positions and peak widths of the two visible absorption bands as fixed from absorption measurements, we could determine the optical dispersion of the film material in $1.3-6 \mathrm{eV}$ energy region. A direct band gap of chromia film grown in these conditions was $3.2 \mathrm{eV}$, the other also direct absorption band with a gap of $5.15 \mathrm{eV}$ was found situated in UV.

K e y w ord s: $\mathrm{Cr}_{2} \mathrm{O}_{3}$, ellipsometry, spectrophotometry, dispersion, band gap
\end{abstract}

\section{Introduction}

Chromium oxide $\left(\mathrm{Cr}_{2} \mathrm{O}_{3}\right)$ has a wide range of applications - catalysts, wear-resistance materials, digital recording systems, materials for thermal protection [1]. Because of its optical properties, it may be used as a dye or pigment colorant material or as an electrochromic material $[1,2]$. It is also used as a component in phaseshifting masks [3] or as a template for growth of the crystalline $\alpha-\mathrm{Al}_{2} \mathrm{O}_{3}[4,5]$. Our goal, too, was to discuss the conditions of alumina growth on chromia template, but this was not possible without knowing the properties of template layer, beforehand.

The atomic layer deposited (ALD) $\mathrm{Cr}_{2} \mathrm{O}_{3}$ films under investigation were grown on $\mathrm{SiO}_{2}$ (fused silica) and $\mathrm{Si}(100)$ substrates using chromyl chloride $\left(\mathrm{CrO}_{2} \mathrm{Cl}_{2}\right)$ and $\mathrm{CH}_{3} \mathrm{OH}$ as precursors [6]. ALD is a process based on saturation of the chemical absorption processes on a surface. The films were deposited using a homemade reactor with optical control of the process [7] using different number of pulses. The process sequence was $0.2-2-2-2 \mathrm{~s}$ (chromyl chloride pulse $-\mathrm{N}_{2}$ purge - methanol pulse $\mathrm{N}_{2}$ purge) at $420^{\circ} \mathrm{C}$ substrate temperature. The obtained films on $\mathrm{SiO}_{2}$ had an incubation phase (number of cycles without recorded growth) of about 20 cycles at this temperature and eskolaite phase was detected already after 25 pulses on $\mathrm{Si}(100)$ using Reflection High-Energy Electron Diffraction and from 200 cycles up using Raman spectroscopy. The chromia films obtained at these conditions on the (1-102) sapphire substrates at $375^{\circ} \mathrm{C}$ have been shown to grow epitaxially [8]. The films mean density rose from 4.9 to $5.1 \mathrm{~g} / \mathrm{cm}^{3}$ with rising thickness up to $70 \mathrm{~nm}$ and remained constant at higher thicknesses [6]. The films contained about $0.02 \% \mathrm{Cl}$ and $0.6 \% \mathrm{C}$ as a residue from growth process. A most thick film on silica was grown using a $0.2 \mathrm{~s} \mathrm{CH}_{3} \mathrm{OH}$ pulse at $375^{\circ} \mathrm{C}$, having about the same physical properties.

\section{$2 \mathrm{Cr}_{2} \mathrm{O}_{3}$ optical properties}

Discussing the optical properties of chromia films, much of differing results have been obtained for a material grown in different conditions. For evaporated $\mathrm{Cr}$ films fired in wet $\mathrm{H}_{2}$ at $1175 \mathrm{~K}$ [9] observed four absorption peaks at $600(2.1 \mathrm{eV}), 460(2.7 \mathrm{eV}), 355$ (3.5 $\mathrm{eV})$ and, possibly, $250(5.0 \mathrm{eV}) \mathrm{nm}$. Karlsson [10], too, saw absorption at 2.1, 2.7 and, possibly, at $3.4 \mathrm{eV}$ in oxidized $\mathrm{Cr}$ films. The absorption bands at the positions 2.2 and $3.0 \mathrm{eV}$ in $\mathrm{Cr}_{2} \mathrm{O}_{3}$ were identified as ${ }^{4} A_{2} \longrightarrow{ }^{4} T_{2}$ and ${ }^{4} A_{2} \longrightarrow{ }^{4} T_{1}$ in [2]. A variation of chromia absorption bands positions in compounds due to different surroundings was shown as [11] $13700-14700(1.70-1.82 \mathrm{eV})$, $14400-15220(1.79-1.89 \mathrm{eV}), 16300-19900(2.02-2.47$ $\mathrm{eV})$ and $21700-25600 \mathrm{~cm}^{-1}(2.70-3.17 \mathrm{eV})$ and identified as ${ }^{4} A_{2 \mathrm{~g}} \longrightarrow{ }^{2} E_{\mathrm{g}},{ }^{4} A_{2 \mathrm{~g}} \longrightarrow{ }^{2} T_{1 \mathrm{~g}},{ }^{4} A_{2 \mathrm{~g}} \longrightarrow{ }^{4} T_{2 \mathrm{~g}}$, and ${ }^{4} A_{2 \mathrm{~g}} \longrightarrow{ }^{4} T_{1 \mathrm{~g}}$, correspondingly. [1] showed an absorption band at $300 \mathrm{~nm}(4.1 \mathrm{eV})$ for hydrothermally synthesized $\mathrm{Cr}_{2} \mathrm{O}_{3}$ films having a direct band gap according to Tauc formula $(\alpha h \nu)=A\left(h \nu-E_{\mathrm{g}}\right)^{1 / 2}$ at $3.2 \mathrm{eV}$. For sprayed $\mathrm{Cr}_{2} \mathrm{O}_{3}$ films Misho et al [12] reported a direct energy gap of $3.40 \mathrm{eV}$ and for solvothermal nanocrystalline $\mathrm{Cr}_{2} \mathrm{O}_{3}$ [13] a direct bandgap at 3.6 - $4.0 \mathrm{eV}$. Using UV-VIS spectroscopy, Ivanova et al [14] obtained $\mathrm{Cr}_{2} \mathrm{O}_{3}$ indirect band gap according to a formula $\alpha(\nu)=A\left(h \nu-E_{\mathrm{g}}\right)^{2}$ for carbonyl $\mathrm{Cr}(\mathrm{CO})_{6}$-based amorphous CVD films annealed in air at $400^{\circ} \mathrm{C}$ as $2.95 \mathrm{eV}$, or for crystalline films annealed at $500^{\circ} \mathrm{C}$ as $3.2 \mathrm{eV}$. In the other work [15], the result was a direct band gap of $3.18-3.26 \mathrm{eV}$ before and $3.32-$

*Institute of Physics, University of Tartu, W. Ostwaldi 1, 50411 Tartu, Estonia, aarnek@ut.ee, **Institute of Physics, University of Tartu, W. Ostwaldi 1, 50411 Tartu, Estonia 
$3.38 \mathrm{eV}$ after annealing at $500^{\circ} \mathrm{C}$. Julkarnain et al $[16]$ reported the indirect band at $3.3 \mathrm{eV}$ for evaporated $\mathrm{Cr}$ films annealed to amorphous oxide in air at $570 \mathrm{~K}$, corresponding to a theoretical result of $3.31 \mathrm{eV}$ indirect band gap in [17]. This number was supported by photoluminescence measurements yielding an indirect band gap of $3.33 \mathrm{eV}$, with a refractive index in UV-VIS claimed to lay in a region of $1.3-2.1$. The refractive index in visible region was presented as about $1.6-1.9$ before and about $2.2-2.4$ after annealing. The 2.1 and $2.7 \mathrm{eV}$ absorption bands were also seen over a high base-level absorption. For CVD films grown from chrome acetylacetonate Cheng [18] found an indirect transition with a band gap of $3.0-3.1 \mathrm{eV}$ and an additional $2.75 \mathrm{eV}$ absorption band.

For chromia grown by radio frequency sputtering at different $\mathrm{O}_{2}$ partial pressures, [19] presented the refractive index at $1.96 \mathrm{eV}(633 \mathrm{~nm})$ as $2.20-2.76$ with direct absorption band gap $3.68-3.83 \mathrm{eV}$ from spectroscopic ellipsometry. Tabbal et al [20] analysed the pulsed laser deposited chromia films using ellipsometry and claimed the refractive index as decreasing from 2.85 to 2.51 at 3 $\mathrm{eV}$ energy with substrate temperature rising from 20 to $950^{\circ} \mathrm{C}$ and average grain size growing from 50 to $200 \mathrm{~nm}$. Using the absorption coefficient values from ellipsometric fit, they also obtained the indirect band gap increasing from 3.08 to $3.25 \mathrm{eV}$ with the rise of substrate temperature. Therefore, there are plenty of reasons to reanalyse the optical properties of $\mathrm{Cr}_{2} \mathrm{O}_{3}$ films.

\section{Apparatus}

The ellipsometric spectra of the films on $\mathrm{SiO}_{2}$ and $\mathrm{Si}$ were measured using spectral ellipsometer GES-5E (Semilab Co.) with a microspot option enabling the measurements in $3 \times 0.8 \mathrm{~mm}$ spot. A WinElli II packet was used to analyse the ellipsometric spectra. The transmission and reflection spectra of the films on $\mathrm{SiO}_{2}$ were measured on a spectrophotometer Jasco V-570. The measurements were corrected against the $\mathrm{T}=100 \%$ line for transmission and against a reflection of a reference $\mathrm{Al}$ mirror for reflection spectra. A reference mirror was calibrated comparing it against a good quality fused silica substrate, the silica refraction index data was taken from [21]. The structure of the films was checked by Raman measurements using inVia (Renishaw) confocal spectrometer and by X-Ray Diffraction using reconstructed diffractometer URT-1 (Nauchpribor) [8]. The measurements of the samples surface quality were performed using Atomic Force Microscopy with a multimode AFM Autoprobe CP II (Veeco). All images were recorded in non-contact mode using HA-HR (NT-MDT) series cantilevers under ambient conditions. The Gwyddion free software ver. 2.44 (Czech Metrology Institute) was employed for the image processing using the first-order flattening for background slope removal. Where necessary, the contrast and brightness were adjusted.

\section{Results and Discussion}

Figure 1 presents a Grazing-incidence XRD spectrum of the two samples of $\mathrm{Cr}_{2} \mathrm{O}_{3}$ films grown on $\mathrm{SiO}_{2}$ demonstrating a polycrystalline structure of the film material, with indicated eskolaite reflexes. To get the information about film optical properties, the $\mathrm{Cr}_{2} \mathrm{O}_{3}$ films grown on $\mathrm{SiO}_{2}$ and $\mathrm{Si}$ substrates were analysed by spectral ellipsometry first. To model chromia dispersion, a TaucLorentz dispersion was used as

$$
\varepsilon_{i}(E)=\frac{A E_{0} C\left(E-E_{\mathrm{g}}\right)^{2}}{\left(E^{2}-E_{0}^{2}\right)^{2}+C^{2} E^{2}} \frac{1}{E}
$$

if $E>E_{\mathrm{g}}$, else $\varepsilon_{i}(E)=0$ with additional Lorentz absorption bands as

$$
\begin{aligned}
& \varepsilon_{r}=\frac{A \lambda^{2}\left(\lambda^{2}-\lambda_{0}^{2}\right)}{\left(\lambda^{2}-\lambda_{0}^{2}\right)^{2}+\Gamma^{2} \lambda^{2}} \\
& \varepsilon_{i}=\frac{A \lambda^{3} \Gamma}{\left(\lambda^{2}-\lambda_{0}^{2}\right)^{2}+\Gamma^{2} \lambda^{2}}
\end{aligned}
$$

Here, $E_{0}, E_{\mathrm{g}}, C, A, \Gamma$ and $\lambda_{0}$ are free parameters for Tauc-Lorentz dispersion law. To characterize a quality of fit, a correlation function as $R^{2}=$

$$
\begin{aligned}
& 1-\frac{1}{2} \frac{\sum_{j=1}^{N}\left[\tan \Psi_{\mathrm{ex}}\left(E_{j}\right)-\tan \Psi_{\mathrm{cal}}\left(E_{j}\right)\right]^{2}}{\sum_{j=1}^{N}\left[\tan \Psi_{\mathrm{ex}}\left(E_{j}\right)-M E A N\left(\tan \Psi_{\mathrm{ex}}\left(E_{j}\right)\right)\right]^{2}} \\
& -\frac{1}{2} \frac{\sum_{j=1}^{N}\left[\cos \Delta_{\mathrm{ex}}\left(E_{j}\right)-\cos \Delta_{\mathrm{cal}}\left(E_{j}\right)\right]^{2}}{\sum_{j=1}^{N}\left[\cos \Delta_{\mathrm{ex}}\left(E_{j}\right)-M E A N\left(\cos \Delta_{\mathrm{ex}}\left(E_{j}\right)\right)\right]^{2}}
\end{aligned}
$$

was used. No good fits could be obtained using this approach. Addition of a rough overlayer with a free parameter for film porosity into a model improved a situation, but not to an extent of having dependable results with $R^{2}>0.97$. The porosity was taken into account as a Bruggeman mix of the main layer properties and the air. A reason for this failure seems to be a big number of the free parameters in the dispersion model (film thickness, 5 parameters for Tauc-Lorentz model, 6 parameters if 2 additional absorption bands were included, and 2 parameters to describe a roughness). Therefore, we needed a pre-characterization of the samples.

To obtain more information about the films optical properties, the transmission and reflection spectra of $\mathrm{Cr}_{2} \mathrm{O}_{3}$ films grown using a different number of ALD pulses on fused silica were recorded. The results for these samples after correction against the transmission $(T)$ values of the spectrophotometer without a sample and reflectance $(R)$ after calibrating of a reference mirror are presented on Fig. 2 and Fig. 3. In the visible region, the interference effects are seen. A number of ALD growth cycles for each film is marked. The influence of growing roughness on reflection is seen in UV and a number of ALD growth cycles for each film is marked. Using these 


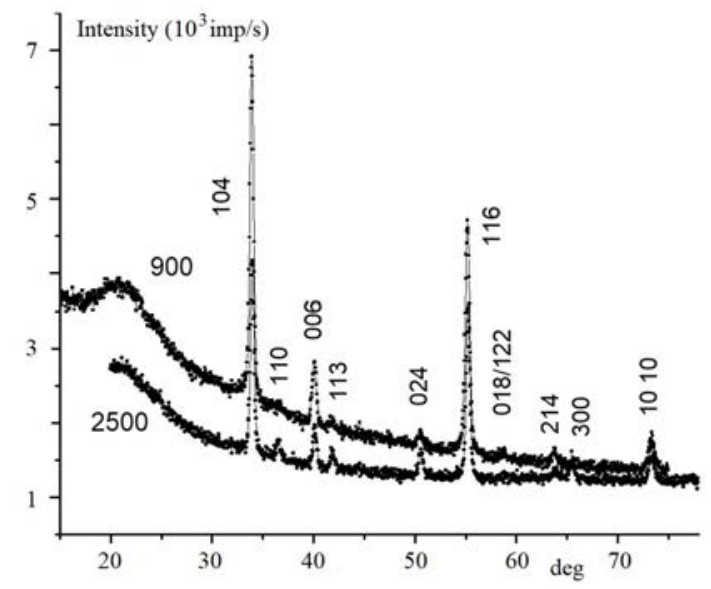

Fig. 1. The GIXRD spectra of the $\mathrm{Cr}_{2} \mathrm{O}_{3}$ films grown using 900 and 2500 ALD pulses

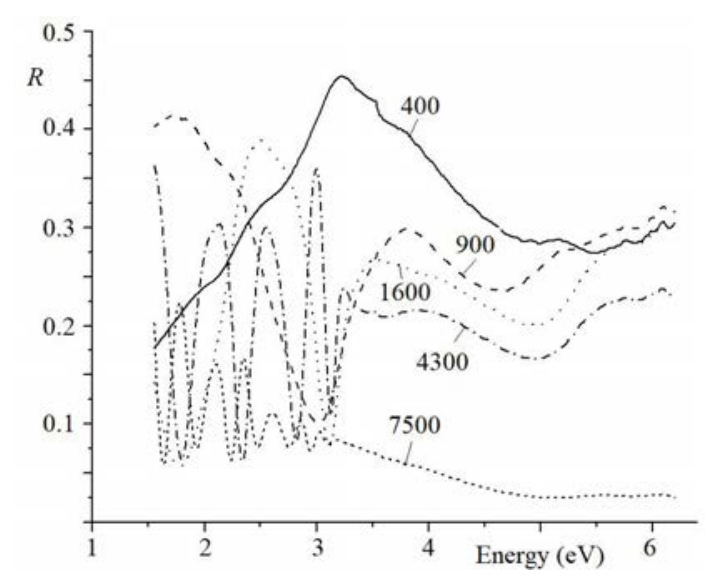

Fig. 3. The reflection spectra of $\mathrm{Cr}_{2} \mathrm{O}_{3}$ films on silica substrates

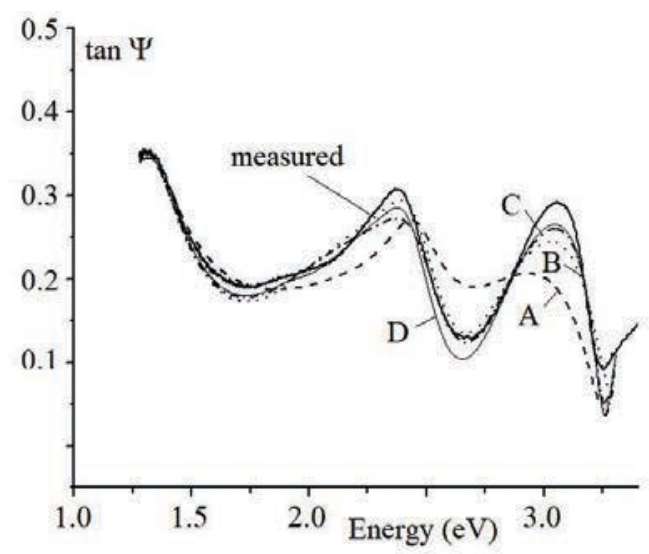

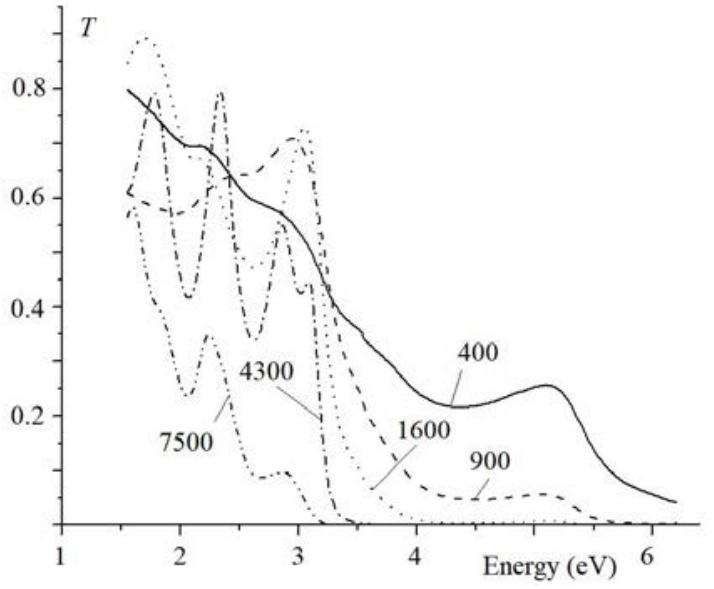

Fig. 2. The transmission spectra of $\mathrm{Cr}_{2} \mathrm{O}_{3}$ films on silica substrates

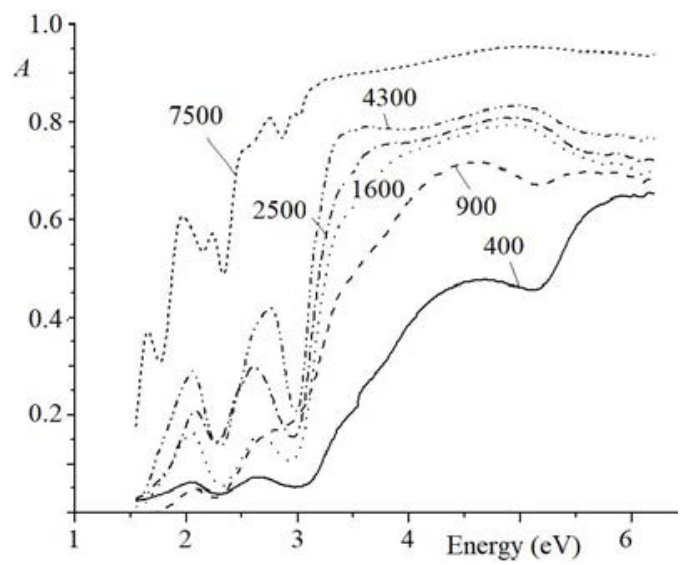

Fig. 4. The absorption spectra of $\mathrm{Cr}_{2} \mathrm{O}_{3}$ films on silica substrates calculated from the transmission and reflection data

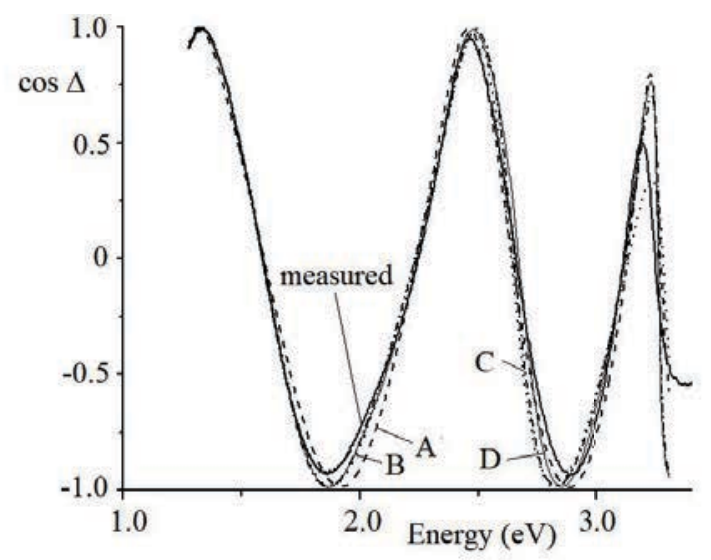

Fig. 5. The ellipsometric spectra of a $\mathrm{Cr}_{2} \mathrm{O}_{3}$ film grown using 2500 ALD cycles

spectra, the absorption dependences (not taking into account a possible scattering) of the films could be calculated as $A=1-R-T$ (Fig. 4).

Figure 4 demonstrates us two already reported absorption bands in visible at the positions 2.0 and $2.6 \mathrm{eV}$ in our case. Their full-widths at half-maximum level (FWHM) were estimated as $0.3 \mathrm{eV}$ from the absorption spectra. In addition, we see an additional structure in the absorp- tion region meaning there is a reason to include into a model one more band in UV. With this information, we proceeded to ellipsometric analysis of the samples again, having fixed the four parameters for the visible absorption bands. Now we had 4 absorption bands for thinner films (400 and 900 cycles) and 3 bands for thicker films where the second blue band in ultraviolet had a negligible influence. The results for a film with thickness of 2500 

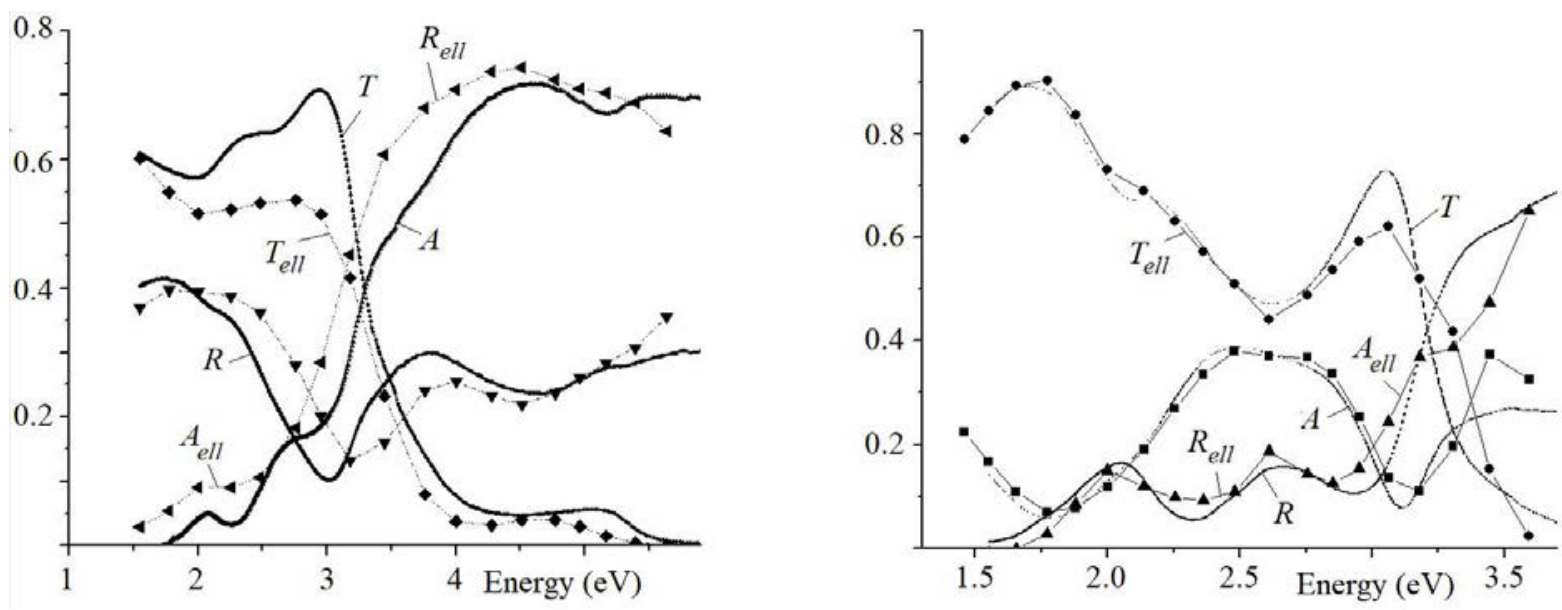

Fig. 6. Reflection, transmission and absorption spectra of $\mathrm{Cr}_{2} \mathrm{O}_{3}$ ALD films on $\mathrm{SiO}_{2}$ grown at 900 and 1600 ALD cycles
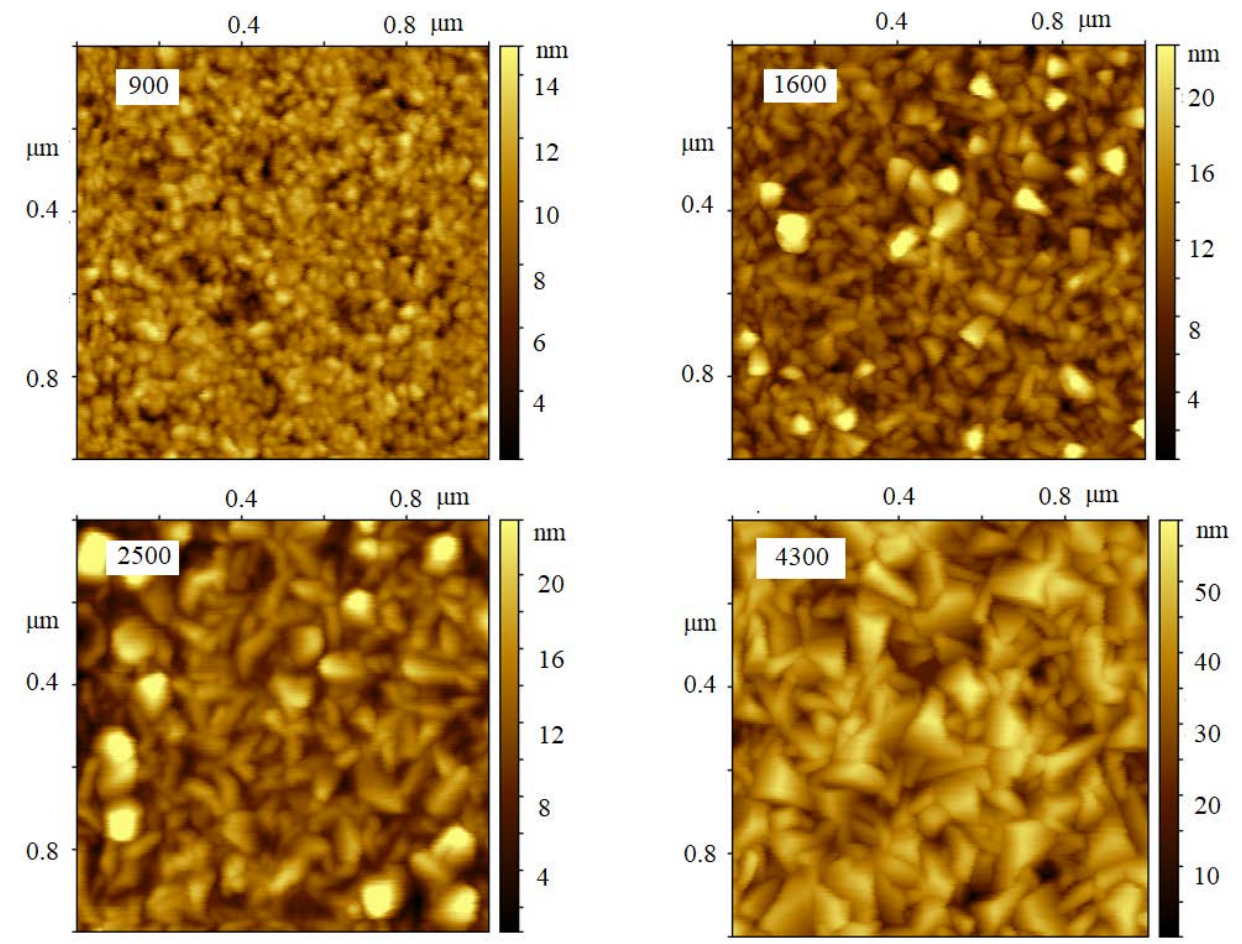

Fig. 7. AFM images (scan area $1 \times 1 \mu \mathrm{m}^{2}$ ) of the $\mathrm{Cr}_{2} \mathrm{O}_{3}$ ALD films with different number of the ALD growth cycles

ALD cycles are shown in Fig. 5. A - fit using 1 absorption band, $\mathrm{B}-1$ absorption band with rough overlayer, $\mathrm{C}$ - 3 absorption bands ( 2 additional bands in visible) and rough overlayer, D - 3 absorption bands and rough overlayer, but the positions and FWHM of the visible bands fixed at fitting. $\mathrm{R}^{2}$ values for the fits were $0.862,0.960$, 0.967 and 0.969 , accordingly.

To crosscheck the applicability of the ellipsometric results the dispersion parameters of the $\mathrm{Cr}_{2} \mathrm{O}_{3}$ films from ellipsometry were used to calculate the spectrophotometric parameters of the films on fused silica according to well-known interference formulae

$$
\begin{aligned}
& r=\frac{r_{21}+r_{10} \exp \left(-2 i \delta_{1}\right)}{1+r_{21} r_{10} \exp \left(-2 i \delta_{1}\right)} \\
& t=\frac{t_{21} t_{10} \exp \left(-2 i \delta_{1}\right)}{1+r_{21} r_{10} \exp \left(-2 i \delta_{1}\right)}
\end{aligned}
$$

where $r_{21}, r_{10}, t_{21}, t_{10}$ are the Fresnel coefficients for light entering from medium 2 to medium 1 , and from medium 1 to medium 0 , accordingly, and $\delta_{1}=2 \pi n d_{1} / \lambda$ is a phase thickness of medium 1 . The dispersion of the fused silica substrate was taken as in [21] and the properties of the rough overlayer as obtained from ellipsometric fit. The results for the films with thicknesses of 900 and 1600 ALD cycles are presented in Fig. 6, where are: lines measured spectra, big marks - values calculated from the modelled ellipsometric spectra taking into account the absorption bands in visible region and rough overlayer. The best coincidence was obtained for a film with thickness of 1600 ALD cycles, which was the thinnest of those opaque in UV region and on a same time had still low thickness of a rough layer. Therefore, we propose that the structure of chromia film in the absorption region is more complicated 
Table 1. Measured ALD $\mathrm{Cr}_{2} \mathrm{O}_{3}$ films sublayer thickness values

\begin{tabular}{lccc}
$\begin{array}{l}\text { Number of } \\
\text { ALD cycles }\end{array}$ & $\begin{array}{c}\text { Main } \\
\text { layer } \\
(\mathrm{nm})\end{array}$ & $\begin{array}{c}\text { Rough } \\
\text { overlayer } \\
(\mathrm{nm})\end{array}$ & $\begin{array}{c}\text { AFM } \\
\text { roughness } \\
(\mathrm{nm})\end{array}$ \\
\hline 400 & 33 & - & - \\
900 & 62 & 10 & 1.5 \\
1600 & 138 & 4 & 2 \\
2500 & 210 & 10 & 3.5 \\
4300 & 412 & 13 & 6 \\
7500 & 811 & 16 & - \\
\hline
\end{tabular}

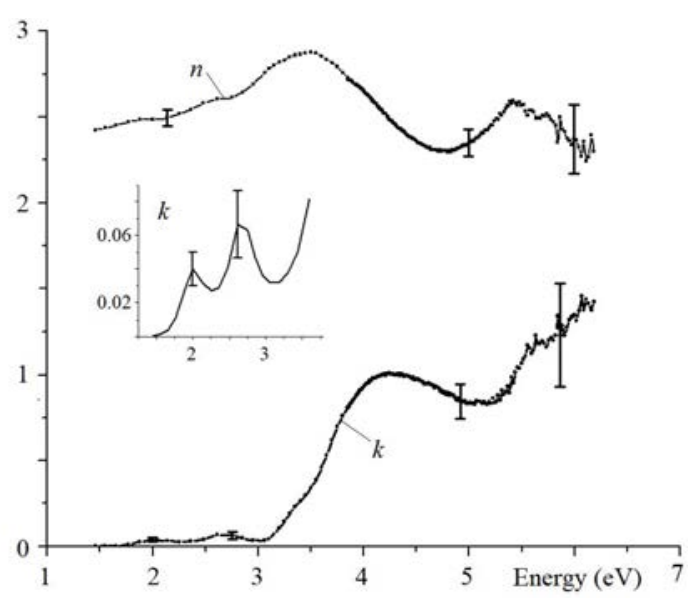

Fig. 8. Optical dispersion of the 1600 cycle $\mathrm{ALD} \mathrm{Cr}_{2} \mathrm{O}_{3}$ film on silica with error margins

than our model with only two Lorentz absorption bands. In addition, it is worth to mark that for two thinnest films we obtained parallel extraneous solutions with better fit quality, but nonphysical properties.

The thickness values of the main and rough layer as obtained from ellipsometric and AFM measurements for our films are given in Tab. 1.

The roughness is caused by crystallization of material during the growth process; the rough layer seems to be partly filled with new material during growth. For a 900 cycles sample, rough layer thickness is probably an artefact of modelling. For 400 cycles, no rough layer was prerequisite to get a good fit.

The optical dispersion in the UV absorption region was calculated from a pseudoellipsometric function

$$
\frac{n-i k}{n_{0}}=\sin \varphi \sqrt{1+\left(\frac{1-\rho}{1+\rho}\right)^{2} \tan ^{2} \varphi}
$$

of the $\mathrm{Cr}_{2} \mathrm{O}_{3}$ films in their opacity region. Here, $n_{0}=1$ is the refractive index of air, $\varphi$ the light incidence angle at measurement, and $\rho=r^{\mathrm{p}} / r^{\mathrm{s}}$ a ratio of $\mathrm{p}$ - and s-polarized reflected light amplitudes. The ellipsometric fit and the pseudoellipsometric function did not correspond to each other in the transition region of $3.3-3.8 \mathrm{eV}$, where the real absorption dependence was already different from the Tauc-Lorentz model, and pseudoellipsometric function already influenced by an advent of transmission. A smooth curve to connect both parts was built there. A result is presented on Fig. 8. The error margins were found as a dispersion of calculation results over all six samples.

To specify the characteristics of a band gap for $\mathrm{Cr}_{2} \mathrm{O}_{3}$, one needs to measure the onset of absorption near to the transmissive region. A formula for this was derived by Moss [22] as

$$
T=\frac{(1-R)^{2}\left(1+\frac{k^{2}}{n^{2}}\right)}{\left(e^{\beta}-R e^{-\beta}\right)+4 R \sin ^{2}(\delta+\varphi)},
$$

where $\beta=2 \pi k d / \lambda$, and $\varphi=\arctan \left(2 k /\left(n^{2}+k^{2}-1\right)\right)$. Only in a situation, where the interferential bands are not resolved more, $k^{2}<<n$, and $\alpha d=2 \beta>>R^{2}$, we obtain better known Moss formula to find an absorption coefficient as

$$
T=(1-R)^{2} e^{-\alpha d}
$$

Here, $T$ and $R$ are the transmission and reflection values of a sample, $\alpha$ is the absorption coefficient, and $n-$ $i k$ is a complex refractive index. The $R$ and $T$ have been measured for chromia samples on fused silica, film thickness value $d$ we obtain from ellipsometric modelling. However, as our samples are clearly too thin to be void of the interference, this formula is not applicable in its pure form.

The values of absorption coefficient were calculated according to Moss formula from measured spectrophotometric spectra only. The results are presented on Fig. 9 . We see that in the visible region the absorption index values of the material lay below zero line. A reason for this is the interference structure we have not taken into account. To correct it in the first approximation, we proceed from an assumption we should have the absorption approximately zero in visible region outside the two absorption bands. Therefore, we can draw a line through the absorption minima of our results and perform a linear transformation of the absorption results so that absorption in the VIS region moves approximately on a zero line. This procedure is demonstrated on Fig. 10. The results for a direct transition model for our samples without and with correction are presented on Fig. 11 and in Table 2. We get a value of $3.2 \mathrm{eV}$ as a band gap for direct transition in $\mathrm{Cr}_{2} \mathrm{O}_{3}$. The thickest film has lower band gap due to defects or possible new structures arising during film growth seen also on Fig. 2. Another possible reason may be the different growth conditions (shorter chromyl chloride pulse) during growth. Good coincidence of the values allows us to assume that the obtained band gap value is reliable. 


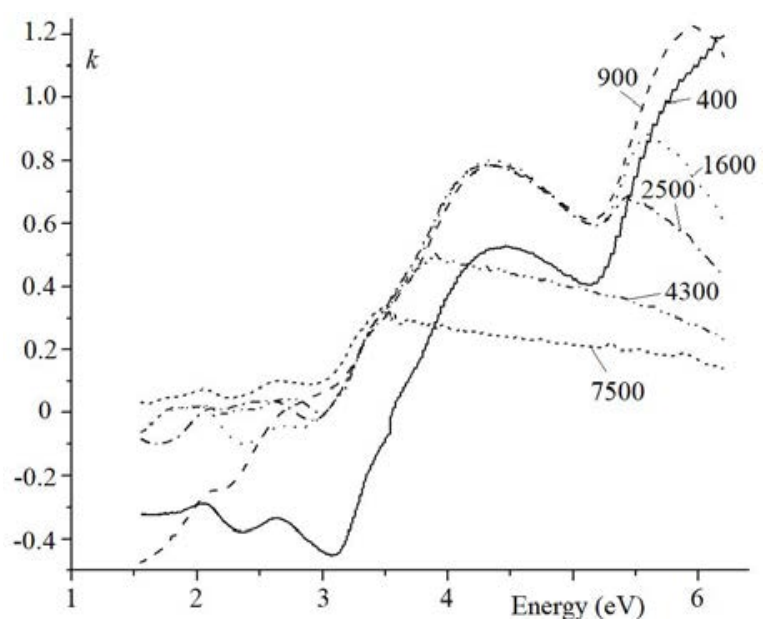

Fig. 9. The absorption index spectra of $\mathrm{Cr}_{2} \mathrm{O}_{3}$ ALD films calculated from Moss formula. The number of ALD cycles of each film is marked.

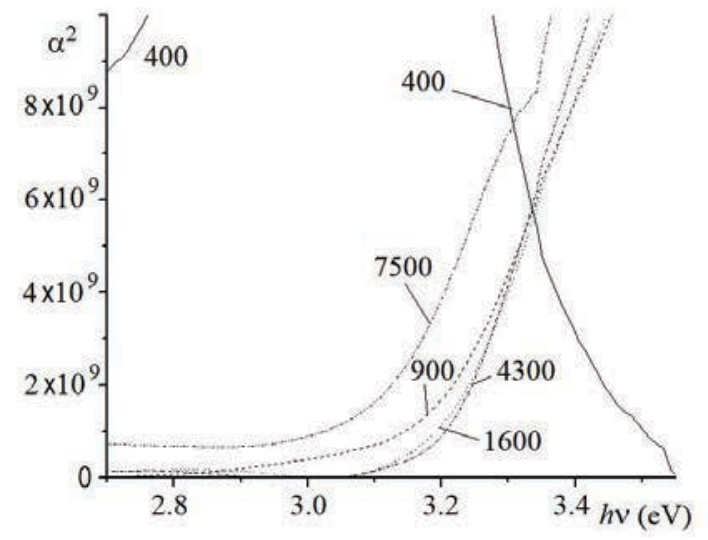

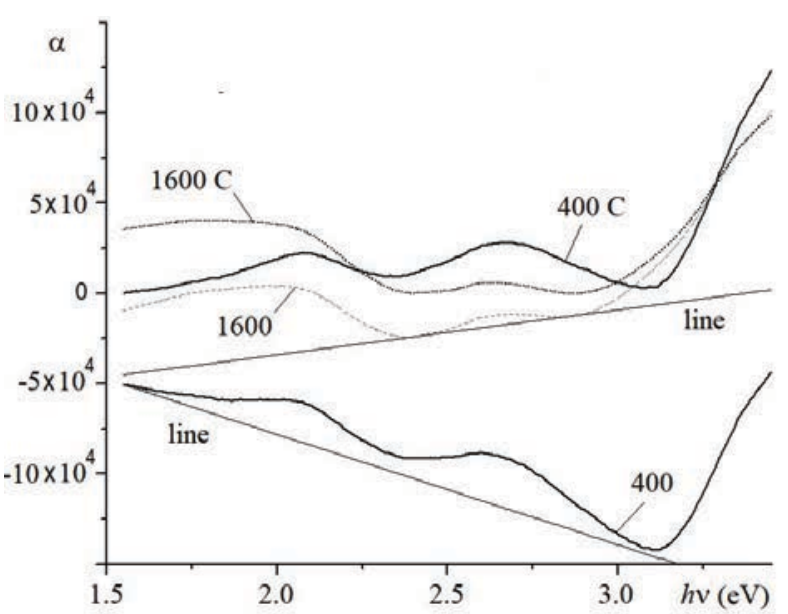

Fig. 10. Correction procedure for thin films in spectral region with interference effects. 400 and 1600 absorption spectra for $\mathrm{Cr}_{2} \mathrm{O}_{3}$ films with given number of ALD cycles, $400 \mathrm{C}$ and $1600 \mathrm{C}-$ the spectra after procedure where a line drawn through their minima in visible region was linearly transformed into an $\alpha=0$ line.

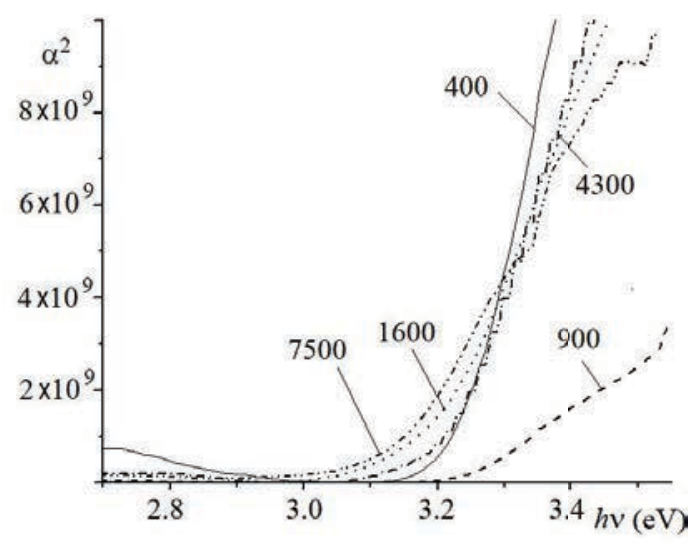

Fig. 11. The absorption spectra for direct gap model according to Moss model (left) and after correction (right). A feature claiming to be high absorption in visible for a thinnest film (400 ALD cycles) at left is almost levelled after correction at right.

Table 2. The absorption edge values obtained from Fig. 11

\begin{tabular}{lcc}
$\begin{array}{l}\text { Number of } \\
\text { ALD cycles }\end{array}$ & $\begin{array}{c}\text { Moss formula } \\
(\mathrm{eV})\end{array}$ & $\begin{array}{c}\text { Corrected spectrum } \\
(\mathrm{eV})\end{array}$ \\
\hline 400 & - & 3.2 \\
900 & 3.2 & 3.2 \\
1600 & 3.2 & 3.2 \\
2500 & 3.2 & 3.2 \\
4300 & 3.2 & 3.2 \\
7500 & 3.1 & 3.15 \\
\hline
\end{tabular}

Additional to the main absorption band, we found the other transition in thinner films on Figs. 4 and 12. Thicker samples are already opaque in UV region and their absorption values saturate as they have been calculated from a noise signal of our spectrophotometer really. This second, also direct transition has a band gap of 5.1 $\mathrm{eV}$ and a maximum placed higher than $6 \mathrm{eV}$. Using Diffuse Reflection Electronic Spectra (DRES) measurements for $\mathrm{CrO}_{\mathrm{x}} / \mathrm{Al}_{2} \mathrm{O}_{3}$ catalysts, [23] saw this absorption band at $280 \mathrm{~nm}(4.4 \mathrm{eV})$. Marinova et al [2] assign a first UV absorption band in $\mathrm{Cr}_{2} \mathrm{O}_{3}$ to $\mathrm{Cr}^{3+}$ spin-allowed transition ${ }^{4} A_{2} \longrightarrow{ }^{4} T_{1}$ (proceeding from ${ }^{4} \mathrm{P}$ state of a free ion). Proceeding from this, we propose that our high-energy band corresponds to transition ${ }^{4} A_{2} \longrightarrow{ }^{2} A_{2}\left({ }^{2} F\right)$ from Tanabe-Sugano diagram [24].

There exists another version for an origin of the absorption band at $229 \mathrm{~nm}(5.4 \mathrm{eV})$ [25]. Here, using Diffuse Reflectance Spectroscopy for $\mathrm{Cr} / \mathrm{Al}_{2} \mathrm{O}_{3}$ catalysts, this band was assigned to solid $\mathrm{K}_{2} \mathrm{CrO}_{4}$ or $\mathrm{K}_{2} \mathrm{Cr}_{2} \mathrm{O}_{7}$ being caused by $\mathrm{Cr}^{+6}$-ion. For the same material, Puurunen [26] sees $\mathrm{Cr}^{6+}$ band at $26000 \mathrm{~cm}^{-1}(3.22 \mathrm{eV})$. As the positions of the bands are dependent on surrounding atoms, we would see in our transmission measurements yielding $\mathrm{Cr}_{3} \mathrm{O}_{3}$ absorption band at $2.6 \mathrm{eV}$, its position in [26, Fig. 4] conditions at $23000 \mathrm{~cm}^{-1}(2.85 \mathrm{eV})$. Then, $\mathrm{Cr}^{6+} \mathrm{red}$ band for our $\mathrm{Cr}_{2} \mathrm{O}_{3}$ material should be situated at about $2.97 \mathrm{eV}$. However, we do not see such band. Neither did a check of our film using the X-ray Photoelectron Spectroscopy reveal any structure at about $579.5 \mathrm{eV}$ binding 


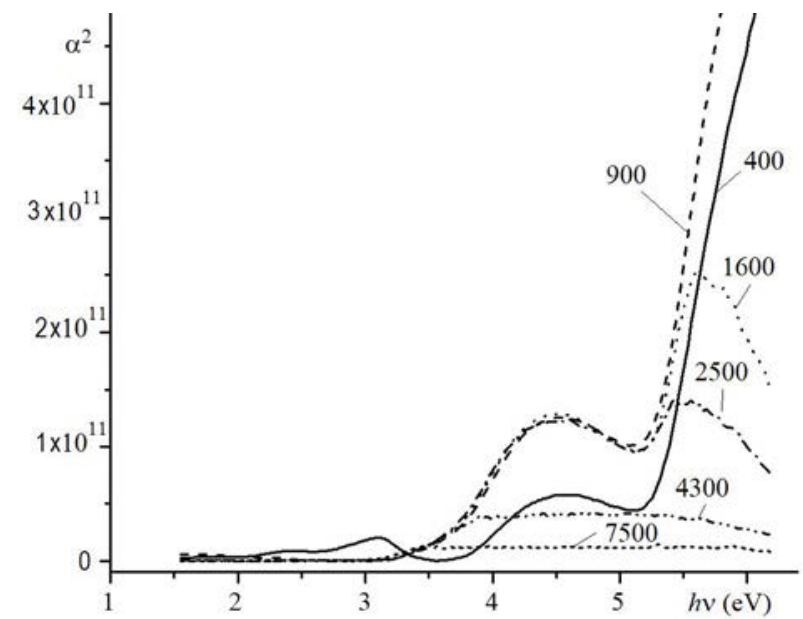

Fig. 12. Absorption coefficient dependence of the ALD $\mathrm{Cr}_{2} \mathrm{O}_{3}$ films of different thickness. The square dependence was chosen to demonstrate a direct nature of the second band in UV.

energy for $\mathrm{Cr}^{6+}$ ion $[27,28]$ so we exclude existence of this feature for our $\mathrm{Cr}_{2} \mathrm{O}_{3}$ films.

\section{Conclusions}

$\mathrm{Cr}_{2} \mathrm{O}_{3}$ films have number of absorption bands in ultraviolet-visible region creating problems in film ellipsometric analysis. A rising number of parameters needed to characterize film dispersion model creates correlations between parameters and makes finding of proper structure problematic. To overcome this problem we derived the absorption spectra of the polycrystalline eskolaite ALD films grown on fused silica proceeding from transmission and reflection dependencies of the films having different thickness values. In this way, we could exclude the interference effects and take the positions and peak widths of the visible bands into account as fixed parameters. This approach furnished us with a possibility to reanalyse the measured spectra and find better solutions. As the films had different roughness values, they were analysed independently and the results checked by recalculation of the spectrophotometric spectra according to ellipsometry results. A best coincidence was achieved for a film with 1600 growth cycles (142 nm). Using ellipsometric modelling in VIS region and pseudoellipsometric function in UV allowed us to find film dispersion from 1.3 to $6 \mathrm{eV}$. A reliability of the obtained dispersion parameters was estimated from the dispersion of the refractive and absorption index values between the different samples.

The absorption spectra were analysed using a Moss absorption formula. As this formula is not correct for thin films, we used both the Moss values and the absorption results after a linear correction based on transmissive region results. The coinciding results show that obtained absorption gap values are dependable for our material. Polycrystalline chromia with eskolaite structure has a direct band gap of $3.2 \mathrm{eV}$. Farther in ultraviolet there is a second absorption band overlapping with the first one and having a maximum at about $6 \mathrm{eV}$. Formerly, the absorption caused by $\mathrm{Cr}^{6+}$ ion was claimed to exist in UV region, but in our case the absorption band we see originates from $\mathrm{Cr}^{3+}$ ion.

\section{Acknowledgements}

The authors gratefully acknowledge a support from Estonian Ministry of Education and Research (Institutional Support Project IUT2-24) and European Regional development Fund (Centre of Intelligence Project TK141).

\section{REFERENCES}

[1] M. M. Abdullah, F. M. Rajab, and Saleh M. Al-Abbas, "Structural and optical characterization of $\mathrm{Cr}_{2} \mathrm{O}_{3}$ nanostructures: Evaluation of its dielectric properties", AIP Advances, vol. 4, $027121,2014$.

[2] Y. Marinova, J. M. Hohemberger, E. Cordoncillo, P. Escribano, J. B. Carda, "Study of solid solutions, with perovskite structure, for application in the field of ceramic pigments", J. Europ. Ceram. Soc., vol. 23, pp. 213-220, 2003.

[3] F. D. Lai, L. A. Wang, "Design of $\left(\mathrm{ZrO}_{2}\right)_{x} /\left(\mathrm{Cr}_{2} \mathrm{O}_{3}\right)_{y}$ $/\left(\mathrm{Al}_{2} \mathrm{O}_{3}\right)_{1-\mathrm{x}-\mathrm{y}}$ superlattices for high transmittance APSM at 193 nm wavelength", Microelectr. Engin., vol. 61-62, pp. 219-226, 2002.

[4] T. Kohara, H. Tamagaki, Y. Ikari, H. Fujii, "Deposition of $\alpha-\mathrm{Al}_{2} \mathrm{O}_{3}$ hard coatings by reactive magnetron sputtering", Surf. Coat. Technol., vol. 185, pp. 166-171, 2004.

[5] A. Khanna, D. G. Bhat, E. A. Payzant, "Growth and characterization of chromium oxide thin films prepared by reactive ac magnetron sputtering", J. Vac. Sci. Technol., vol. A24, pp. 1870-1877, 2006.

[6] A. Tarre, J. Aarik, H. Mändar, A. Niilisk, R. Pärna, R. Rammula, T. Uustare, A. Rosental, V. Sammelselg, "Atomic layer deposition of $\mathrm{Cr}_{2} \mathrm{O}_{3}$ thin films: Effect of crystallization on growth and properties", Appl. Surf. Sc., vol. 254, pp. 5149-5156, 2008.

[7] A. Niilisk, A. Rosental, A. Gerst, V. Sammelselg, T. Uustare, "Atomic-scale optical monitoring of the initial growth of $\mathrm{TiO}_{2}$ thin films", Smart Optical Inorganic Structures and Devices. Proceedings of SPIE, vol. 4318, pp. 72-77, 2001.

[8] H. Mändar, T. Uustare, J. Aarik, A. Tarre, A. Rosental, "Characterization of asymmetric rhombohedral twin in epitaxial $\alpha-\mathrm{Cr}_{2} \mathrm{O}_{3}$ thin films by X-ray and electron diffraction", Thin Solid Films, vol. 515, pp. 4570-4579, 2007.

[9] R. E. Kirby, E. L. Garwin, F. K. King, and A. R. Nyaiesh, "Surface properties of $\mathrm{Cr}_{2} \mathrm{O}_{3}$ ", J. Appl. Phys., vol. 62, pp. 1400-1405, 1987.

[10] B. Karlsson and C. G. Ribbing, "Optical constants and spectral selectivity of stainless steel and its oxides", J. Appl. Phys., vol. 53(9), pp. 6340-6346, 1982.

[11] R. S. Pavlov, V. B. Marzá and J. B. Carda, "Electronic absorption spectroscopy and colour of chromium-doped solids", J. Mater. Chem., vol. 12, pp. 2825-2832, 2002.

[12] R. H. Misho, W. A. Murad, G. H. Fattahallah, "Preparation and optical properties of thin films of $\mathrm{CrO}_{3}$ and $\mathrm{Cr}_{2} \mathrm{O}_{3}$ prepared by the method of chemical spray pyrolysis", Thin Solid Films, vol. 169(2), pp. 235-239, 1989.

[13] K. Anandan, and V. Rajendran, "Chromia $\left(\mathrm{CR}_{2} \mathrm{O}_{3}\right)$ Nanoparticles Synthesized by using $\mathrm{CRO}_{3}$ via the facile Solvothermal Process and their Optical Properties", Intern. J. Engin. Sciences Res. Technol., vol. 6(7), pp. 916-921, 2017. 
[14] T. Ivanova, M. Surtchev, and K. Gesheva, "Characterization of CVD Chromium Oxide Thin Films", Phys. Stat. Sol. A, vol. 184(2), pp. 507-513, 2001.

[15] T. Ivanova, K. Gesheva, A. Cziraki, A. Szekeres and E. Vlaikova, "Structural transformations and their relation to the optoelectronic properties of chromium oxide thin films", J. Phys.: Conf. Ser., vol. 113, 012030, 2008.

[16] M. Julkarnain, J. Hossain, K. S. Sharif, and K. A. Khan, "Optical properties of thermally evaporated $\mathrm{Cr}_{2} \mathrm{O}_{3}$ thin films", Can. J. Chem. Engineering \& Technology, vol. 3(4), pp. 81-85, 2012.

[17] Y. Guo, S. J. Clark and J. Robertson, "Electronic and magnetic properties of $\mathrm{Ti}_{2} \mathrm{O}_{3}, \mathrm{Cr}_{2} \mathrm{O}_{3}$, and $\mathrm{Fe}_{2} \mathrm{O}_{3}$ calculated by the screened exchange hybrid density functional", J. Phys.: Condens. Matt., vol. 24, 325504, 2012.

[18] Ch.-S. Cheng, H. Gomi, and H. Sakata, "Electrical and Optical Properties of $\mathrm{Cr}_{2} \mathrm{O}_{3}$ Films Prepared by Chemical Vapour Deposition", Phys. Stat. Sol. A, vol. 155, pp. 417-425, 1996.

[19] P. Hones, M. Diserens, F. Lévy, "Characterization of sputter-deposited chromium oxide thin films", Surf. Coat. Technol., vol. 120-121, pp. 277-283, 1999.

[20] M. Tabbal, S. Kahwaji, T. C. Christidis, B. Nsouli, K. Zahraman, "Pulsed laser deposition of nanostructured dichromium trioxide thin films", Thin Solid Films, vol. 515, pp. 1976-1984, 2006.

[21] I. H. Malitson, "Interspecimen Comparison of the Refractive Index of Fused Silica", J. Opt. Soc. Am., vol. 55(10), pp. 1205-1209, 1965.

[22] T. S. Moss, G. J. Burrell, B. Ellis, Semiconductor Opto-Electronics, Butterworth and Co (Publishers) Ltd., pp. 19, 1973.

[23] A. A. Rastorguev, M. G. Baronskiy, N. A. Zaitseva, L. A. Isupova, A. I. Kostyukov, T. V. Larina, N. A. Pakhomov, and
V. N. Snytnikov, "Photoluminescence Properties of Microspherical Alumina-Chromium Catalyst", Inorganic Materials: Applied Research, vol. 5(5), pp. 476-481, 2014.

[24] G. K. B. Costa, A. López, I. C. S. Carvalho, O. Nakamura, N. Cella, L. P. Sosman, "Optical and structural properties of chromium impurities in niobium-gallium oxide", Materials Chem. Phys., vol. 148, pp. 764-771, 2014.

25] B. M. Weckhuysen, R. A. Schoonheydt, "Recent progress in diffuse reflectance spectroscopy of supported metal oxide catalysts", Catalysis Today, vol. 49, pp. 441-451, 1999.

[26] R. L. Puurunen and B. M. Weckhuysen, "Spectroscopic Study of the Irreversible Deactivation of Chromia/Alumina Dehydrogenation Catalysts", J. of Catalysis, vol. 210, pp. 418-430, 2002.

[27] M. C. Biesinger, B. P. Payne, A. P. Grosvenor, L W. M. Lau, A. R. Gerson, R. St. C. Smart, "Resolving surface chemical states in XPS analysis of first row transition metals, oxides and hydroxides: Cr, Mn, Fe, Co and Ni", Appl. Surf. Sc., vol. 257, pp. 2717-2730, 2011.

[28] T. C. Kaspar, S. E. Chamberlin, S. A. Chambers, "Surface structure of $\alpha-\mathrm{Cr}_{2} \mathrm{O}_{3}(0001)$ after activated oxygen exposure", Surf. Sc., vol. 618, pp. 159-166, 2013.

Received 19 March 2019

Aarne Kasikov was born April 1957 in Estonia. Obtained MSc (1995) and PhD in Applied Physics (2010) from the University of Tartu, researcher. Interests: vacuum evaporation, thin film interference, ellipsometry.

Margus Marandi was born in Estonia. Obtained MSc (2004) and PhD in Inorganic Chemistry (2011) from the University of Tartu, researcher. Interests: scanning probe microscopy, conducting polymer thin films. 